Aus dem rlinisghen Röntaen- Institute Zürich.

\title{
WARUM BRICHT DER LEBENDE KNOCHEN LEICHTER ALS DER TOTE?
}

\author{
vom \\ H. ZUPPINGER, \\ ZÜRICH.
}



Es ist seit langer Zeit beobachtet, dass der Knochen an der Leiche zum Bruche einer viel grösseren Gewalteinwirkung bedarf als am Lebenden. In der neueren Litteratur müssen die Angaben darüber sehr spärlich sein, da ich beim Suchen danach nicht mehr als einige kurze Andeutungen gefunden habe. Speziell in der chirurgischen Litteratur werden die Festigkeitsverhältnisse des toten Knochens angeführt, ohne dass dabei die Bemerkung gemacht würde, dass beim lebenden Knochen die Verhältnisse sehr abweichende zu sein scheinen. Eine Erklärung für die verschiedene Resistenz des toten und des lebenden Knochens giebt es meines Wissens bis jetzt nicht. Das muss um so eher befremden, als gerade in den letzten dreissig Jahren immer häufiger Versuche gemacht wurden, die verschiedenen Frakturformen experimentell herzustellen. Einem jeden, der solche Versuche macht, muss die grosse Kraft auffallen, die zum Bruche toter Knochen verwendet werden muss, während der nämliche Knochen am Lebenden oft unglaublich leicht einbricht. Ferner kann es einem Experimentator nicht entgehen, dass er leichter eine Bänderzerreissung oder eine Luxation zu stande bringt als einen Knochenbruch, während bekanntlich am Lebenden die Frakturen ungefähr $7 \mathrm{mal}$ häufiger sind als die Luxationen. Ich selber wollte vor 4 Jahren, als ich die Torsionsfrakturen bearbeitete, einen toten Unterschenkel durch Drehung zum Bruche bringen. Der Anatomieabwart erklärte mir von 
vornherein, das sei unmöglich, und in der That konnten wir, zusammen 4 Mann, allerdings den Fuss im Fussgelenk abdrehen, aber ohne dass die Tibia brach. Und wie spielend leicht entsteht die Torsionsfraktur des Unterschenkels am Lebenden! Bei dieser Gelegenheit war ich dann auch erstaunt, zu sehen, dass man eine tote Fibula langsam bis um $60^{\circ}$ biegen kann, ehe sie bricht, während Biegungsfrakturen an der Fibula sogar durch Muskelzug entstehen.

Aber auch am Lebenden hat man häufig die Beobachtung gemacht, dass in der Narkose, tiefem Schlaf, sinnloser Betrunkenheit, ein schweres Trauma eine Fraktur nicht zu stande bringt, während man am Wachenden eine solche bei gleicher Gewalteinwirkung durchaus erwarten müsste.

Es liegt nun kein ersichtlicher Grund vor, warum der Knochen sowohl im lebenden als im toten Zustande nicht den allgemeinen Gesetzen der Festigkeit und der Mechanik überhaupt unterworfen sein sollte. So lange unter dieser Voraussetzung eine Erklärung der vorliegenden Frage möglich ist, kann auf irgend ein geheimnisvolles Agens verzichtet werden. Die Verschiedenheit der Knochenfestigkeit wird bedingt sein müssen entweder durch eine Verminderung oder Vermehrung der Kohäsion, oder dann ist am lebenden Knochen noch eine äussere Kraft wirksam, welche am toten wegfällt.

Es ist in die Augen springend, dass es hauptsächlich drei Umstände sind, die sich beim Eintritt des Todes wesentlich ändern :

1. die Temperatur,

2. der Blutdruck,

3. die Muskelaktion.

1. Die Temperatur könnte insofern in Betracht kommen als allgemein die Temperatursteigerung eine Kohäsionsverminderung bewirkt. Es ist aber nicht wahrscheinlich, dass eine Temperaturabnahme von ca. $30^{\circ}$ eine erhebliche Festigkeits- 
vermehrung bedingt. Durch Anwärmen des kalten, toten Knochens ist auch eine Festigkeitsverminderung nicht hervorzubringen.

2. Dẹr arterielle Druçk, In grösseren Röhrenknochen tritt das Blut ein unter einem Druck von ca. $160 \mathrm{~mm}$ Quecksilber, und tritt aus unter einem Druck von ca. $60 \mathrm{~mm}$ Quecksilber. Dieser Spannungsverlust des Blutes geht auf den Knochen über, das heisst: der lebende Knochen steht unter einem Druck oder unter einer Spannung von $100 \mathrm{~mm}$ Quecksilber, während der tote Knochen keiner solchen Spannung unterworfen ist. Dieser innere Druck bringt nun, da er gleichmässig nach allen Seiten gerichtet ist, keine gegenseitigen Verschiebungen der Elemente zu stande, erzeugt aber eine nicht geringe Oberflächenspannung. Vermöge dieses inneren Druckes besteht in den lebenden Knochen die Tendenz, diejenige Form anzunehmen, die bei gleicher Oberfläche den grössten Inhalt hat, nämlich die Kugelform. Ein Druck von $100 \mathrm{~mm}$ Quecksilber ist natürlich nicht im stande, eine nachweisbare Formveränderung des Knochens herbeizu führen, ein genügend starker Druck aber hätte das Bestreben, einen langen Knochen in der Mitte aufzubläben und schliesslich zu sprengen, ähnlich wie das erbrütete Küchlein die Eischale äquatorial sprengt.

Dieser innere Druck bewirkt, indem er eine Spannung der Oberfäche setzt, eine Einengung der Elastizitätsgrenze, eine grössere Sprödigkeit. Dass er nicht ohne Wirkung ist, zeigt das Verhalten rasch gekühlten Glases, Stahls, Gusseisens; aber auch turgide Pflanzenteile werden brüchig, während welke es' nicht sind. Dass aber speziell der Blutdruck von Einfluss auf die Festigkeit der Gewebe ist, ergiebt sich aus den Biegungsfrakturen des erigierten Penis.

3. Viel wichtiger aber scheint mir der Einfluss der Muskelaktion auf die Entstehung der Knochenbrüche. Es sind über die Kraftentwickelung der Muskeln allgemein recht unbestimmte 
und auch unrichtige Ansichten verbreitet: Und ein Teil der Muskelwirkung wird fast immer ausser acht gelassen; deshalb hier zwei Beispiele:

Es sei beispielsweise das Gewicht eines Armes $5 \mathrm{~kg}$, der Schwerpunkt des gestreckten Armes liege in der Ellenbogenbeuge, $33 \mathrm{~cm}$ vom Mittelpunkt des Humerus-Kopfes entfernt. Dieser Arm werde horizontal nach aussen und vorwärts gehalten, während die Hand, in einem Abstand von $66 \mathrm{~cm}$, von der Mitte des Humerus-Kopfes gemessen, ein Gewicht von $10 \mathrm{~kg}$ trage. Diese beiden Lasten werden erhoben und getragen durch die Muskeln, welche den Schultergürtel heben, und durch den Musculus deltoides, der den Arm im Schultergelenk hebt. Diese beiden Gruppen nun sind nicht neben-, sondern hintereinander geordnet, und es hat der Deltoides für sich allein die beiden Lasten vollständig im Gleichgewicht zu halten, gerade wie bei einer Kette jedes einzelne Glied, ob deren viele oder wenige seien, die angehängte Last zu tragen hat. Der Musculus supraspinatus wird hier vernachlässigt, weil er noch grössere Zahlen ergäbe, und weil er, wie Infraspinatus und Subscapularis zum Gelenkapparat gehören und der Hauptsache nach kontraktile Ligamente - sit venia verbo - sind.

Es ergibt sich für die Wirkungsweise des Deltoides folgende einfache Rechnung, bei der noch angenommen wird, die Insertion des Deltoides sei $11 \mathrm{~cm}$ von der Mitte des Humerus-Kopfes entfernt, und die Zurichtung des Deltoides bilde mit dieser Distanz einen Winkel von $22^{1 / 20}$.

Von vornherein ist einleuchtend, dass nicht die ganze Leistung des Deltoides zur Hebung der beiden Lasten verwendet wird; es ist demgemäss die Zugkraft des Deltoides in zwei Komponenten zu zerlegen: in eine Komponente $R$, welche in die Richtung von der Insertion zum Drehpunkt im Humeruskopfe füllt, und in eine Komponente S, welche an der Insertions- 
stelle senkrecht auf der ersten steht. Alsdann ist in Bezug auf den Drehpunkt im Humeruskopfe

$$
\begin{aligned}
11 \times \mathrm{S} & =33 \times 5 \mathrm{~kg}+66 \times 10 \mathrm{~kg} \\
& =825 \mathrm{~kg} \\
\mathrm{~S} & =\frac{825}{11} \mathrm{~kg}=75 \mathrm{~kg}
\end{aligned}
$$

Die Kraft von $75 \mathrm{~kg}$ wird also dazu verwendet, dem Gewicht des erhobenen, gestreckten Armes und der Last von $10 \mathrm{~kg}$ an der Hand das Gleichgewicht zu halten.

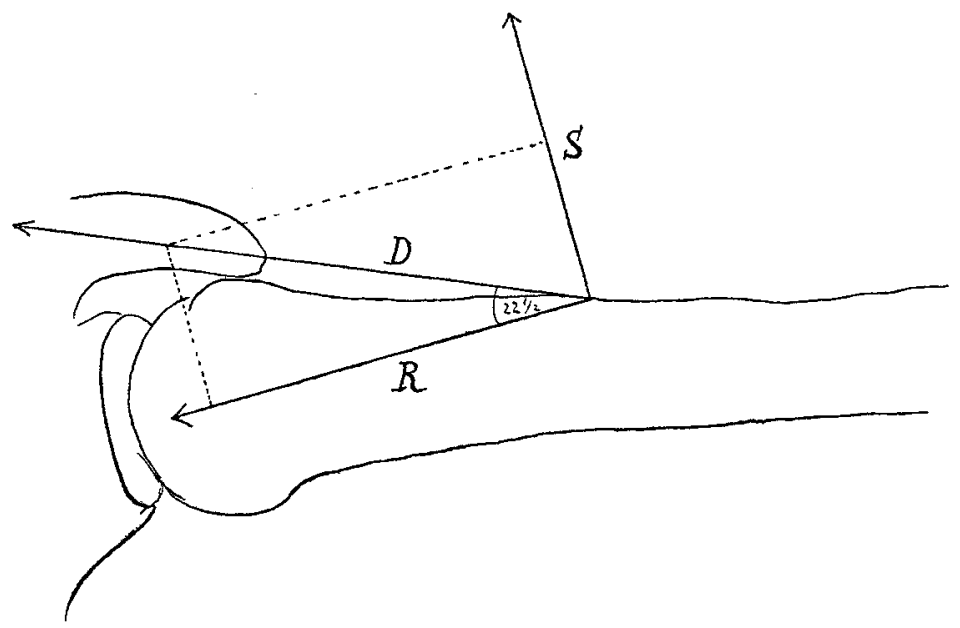

Fig. 1.

Bedeutet nun D die gesamte in Verwendung kommende Zugkraft des Deltoides, so ist aber auch:

$$
\begin{aligned}
& \mathrm{S}=\mathrm{D} \cdot \sin 22^{1 / 2^{\circ}} \\
& \mathrm{R}=\mathrm{D} \cdot \cos 22^{1} / 2^{\circ} .
\end{aligned}
$$

Es ist aber $\cos 22^{1 / 2^{0}} 2^{1 / 2}$ mal so gross, als $\sin 22^{1 / 2^{0}}$, woraus folgt, dass $\quad R=187^{1} / 2 \mathrm{~kg}$

$$
\text { und } \mathrm{D}=202 \mathrm{~kg} \text { ist. }
$$

$\mathrm{R}$ ist aber der Druck, mit welchem das obere Drittel des Humerus vom Deltoides bei diesem keineswegs extremen Beispiel 
gegen die Cavitas glenoides gepresst wird. D andererseits ist ein Zug, der gleichzeitig an der Insertion des Deltoides wirkt.

Das sind bereits recht stattliche Zahlen, und, wie man sieht, ist bereits in diesem Falle die Kraft, mit welcher der Deltoides den Kopf ins Gelenk presst, ein Vielfaches vom Drucke der Luft, der einzig der Trennung der Gelenkflächen entgegenwirk̀t.

Aber das ist noch nicht der ganze Muskeldruck gegen das Schultergelenk; ausser dem Deltoides kommen noch die Gelenkmuskeln Subscapularis, Supra- und Infraspinatus in Aktion und setzen das Caput humeri unter einen noch höheren Druck, und ebenso wirken auf das ganze Os humeri die in einer Schleife angeordneten Extensoren und Flexoren des Ellbogengelenks.

Es findet also bei dem Beispiel eine sehr hohe Druckbelastung des Humerus in seiner. Längsrichtung und eine Anzahl von Zugbelastungen statt, beide durch die Muskelaktion, also nur am Lebenden.

$\mathrm{Z}$ weites Beispiel. Ein Mann von 75 kg Gewicht steht auf den Zehenballen des eines Fusses. Unter welchem Druck steht die Tibia?

Der Zehenstand wird aufrecht erhalten durch Anspannung der Wadenmuskulatur. Diese zieht ihre Insertion am Calcaneus aufwärts; die belastete Tibia drückt im Fussgelenk abwärts. Punctum fixum sind die Capitula metatarsorum. Der Hebelarm der Last ist $=2$, derjenige des Muskelzuges $=3$. Für Gleichgewicht, d. h. Stehen, ist

$$
2 \mathrm{~T}=3 \mathrm{M}
$$

wobei $\mathrm{T}$ den Druck der Tibia auf den Talus, $\mathrm{M}$ den Zug der Wadenmuskulatur bedeutet.

Es zieht aber die Wadenmuskulatur ebenso stark, wie am Calcaneus, auch oben am Uuterschenkel und am Femur, und 
dieser letztere Zug drückt nun hinwieder, ausser der Körperlast von $75 \mathrm{~kg}$, auf die Tibia. Es ist also:

$$
\mathrm{T}=\mathrm{L} \text { (ast) }+\mathrm{M} \text {. }
$$

In obige Gleichung eingesetzt kommt:

$$
2 \mathrm{~L}+2 \mathrm{M}=3 \mathrm{M}
$$

beiderseits $2 \mathrm{M}$ subtrahirt

$$
\mathrm{M}=2 \mathrm{~L}=150 \mathrm{~kg} \text {. }
$$

Dieser Wert oben eingesetzt, gibt:

$$
\mathrm{T}=3 \mathrm{~L}=225 \mathrm{~kg} \text {. }
$$

Beim Zehenstand auf einem Fuss ergibt sich für einen Mann von $75 \mathrm{~kg}$ ein Muskelzug am Ursprung der Wadenmuskulatur und ebenso am Calcaneus von $150 \mathrm{~kg}$ und eine Druckbelastung der Tibia von $225 \mathrm{~kg}$.

Trägt der Mann dazu auf der Schulter einen Sack von $50 \mathrm{~kg}$, so wird die beiderseitige Zugwirkung der Wadenmuskulatur je $250 \mathrm{~kg}$, die Druckbelastung der Tibia $375 \mathrm{~kg}$.

Die beiden Beispiele bedeuten nun allerdings eine namhafte aktive Muskelleistung, doch keineswegs eine höchste Anstrengung. Aber auch ohne Intention einer Muskelarbeit sind am wachenden Lebenden alle Muskeln in mehr oder weniger starker Spannung, die sich zwar schwer bemessen lässt. Doch werden auch diese Spannungen ganz beträchtliche Druck- und Zugwerte bedingen, die am Toten und Bewusstlosen fehlen. Die unwillkürlichen Muskelspannungen bei imminenter Gefahr dürften aber doch denen der beiden Beispiele nicht nachstehen.

Es wäre nun noch nachzuweisen, dass die hier gezeigten Zug- und Druckkräfte, die am Lebenden auf den Knochen einwirken, den Bruch begünstigen. Für Stauchungs-, Knickungsund Abreissungsfrakturen ist das überhaupt nicht nötig, weil eben die Kraft des Traumas für sich allein weniger Wirkung hat, als zusammen mit der gleichgerichteten Kraft des Muskeldruckes und -zuges. Für Biegungs- und Torsionsfrakturen wirken Muskelzug und -druck ebenfalls begünstigend, doch würde der 
Nachweis zu weit führen. Ebenso kann auf den Uuterschied zwischen Druck und Stoss hier nicht eingegangen werden.

Nachdem nun derjenige Muskeldruck und -zug, der sonst einfach ignoriert wird, sich als keineswegs geringfügig, sondern als sehr erheblich herausgestellt hat und derselbe nur beim Lebenden wirkt, beim Bewusstlosen und der Leiche aber wegfällt, so möchte ich ihn als die Hauptursache der grösseren Brüchigkeit des lebenden. Knochens erklären. Dem arteriellen Druck.wird eine untergeordnete Bedeutung nicht abzusprechen sein.

Zum Schlusse sei mir noch eine Bemerkung gestattet. Auf die Berücksichtigung der Druckkomponente des Muskelzuges bin ich geführt worden durch die Beschäftigung mit den Gelenken, und es scheint mir, dass dieselbe in der Gelenktheorie eine nicht unwichtige Rolle spielen wird. Schon aus diesem Grunde wäre es eine dankenswerte Aufgabe, die Muskeln nach dieser Seite hin genauer zu studieren. Aber ausserdem ist es fast undenkbar, dass diese Druckkomponente nicht auch ihre Spur in der Struktur der Spongiosa, in der äusseren Form der Knochen und endlich in Deformitäten hinterlassen sollte; denn jeder Muskel übt einen Zug an Ursprung und Insertion, der in der Richtung nur wenig variiert, und übt einen „Auflager"druck auf das Gelenk aus von fast konstanter. Richtung. Es kommt also nicht einmal so sehr auf gewohnheitsmässige Haltungen und Bewegungen, als auf die Anordnung der Muskulatur an.

Aber auch in der Frakturlehre wird dieser Muskeldruck und -zug von Bedeutung sein. Er wird einige Bruchformen besser erklären; er wird einige Dislokationen verständlicher machen und auch für die Behandlung einige Winke geben. 